\begin{tabular}{|c|l|}
\hline Title & Estimation of Engel Curves from Survey Data with Zero Expenditures \\
\hline Author(s) & Hasegawa, Hikaru; Ueda, Kazuhiro; Mori, Kunie \\
\hline Citation & $\begin{array}{l}\text { Oxford Bulletin of Economics and Statistics, 70(4), 535-558 } \\
\text { https://doi.org/40.1111/.1468-0084.2008.00507.x }\end{array}$ \\
\hline Issue Date & 2008-08 \\
\hline Doc URL & http://hdl.handle.net/2115/47265 \\
\hline Rights & The definitive version is available at www.blackwell-synergy.com \\
\hline Type & article (author version) \\
\hline File Information & OBES70-4535-558.pdf \\
\hline
\end{tabular}

Instructions for use 


\title{
Estimation of Engel Curves from Survey Data with Zero Expenditures*
}

\author{
Hikaru Hasegawa $\dagger$, Kazuhiro Ueda $\ddagger$ and Kunie Mori§ \\ $\dagger$ Graduate School of Economics and Business Administration, Hokkaido \\ University, Sapporo, Japan \\ (e-mail: hasegawa@econ.hokudai.ac.jp) \\ $\ddagger$ Faculty of Economics, Nihon Fukushi University, Okuda, Mihama-cho, \\ Chita-gun, Aichi. 470-3295. Japan \\ (e-mail: ueda@n-fukushi.ac.jp) \\ $\S$ Faculty of Economics, Shimonoseki City University, Shimonoseki, Japan \\ (e-mail: mori-k@shimonoseki-cu.ac.jp)
}

\begin{abstract}
The objective of this article is to propose a Bayesian method for estimating a system of Engel functions using survey data that includes zero expenditures. We deal explicitly with the problem of zero expenditures in the model and estimate a system of Engel functions that satisfy the adding-up condition. Furthermore, using MCMC, we estimate unobservable parameters, including consumption of commodities, total consumption and equivalence scale, and use their posterior distributions to calculate inequality measures and total consumption elasticities.
\end{abstract}

JEL Classification: C11 , D12

KEYWORDS: Bayesian method, generalised entropy measure, Gini coefficient, Markov chain Monte Carlo (MCMC), Working-Leser Engel curves

\section{Introduction}

\footnotetext{
${ }^{*}$ We are grateful to the Institute for Research on Household Economics for providing the micro data of the Japanese Panel Surveys of Consumption. Further, we appreciate the comments of two anonymous referees, professors Christopher Adam (the editor of this journal) and Noriko Hashimoto, and the participants of the seminars held at the Institute for Research on Household Economics and Doshisha University; these comments have significantly improved the article. The work of the first author was supported in part by a Grant-in-Aid for Scientific Research (No.16530137) from the JSPS, while that of the first and second authors was supported in part by a Grant-in-Aid for Scientific Research (No.14653004) from the MEXT.
} 
Household budget surveys are a useful source of data for the estimation of consumer behaviour. However, when using micro data on household expenditure, we often find that expenditure is recorded as zero for certain commodities during the survey period. There are three possible causes for such zero expenditures. ${ }^{1}$ Firstly, certain consumers may prefer not to purchase or consume certain commodities, for example alcohol and tobacco. In this case, consumers are at a corner solution. Secondly, although the commodity is actually consumed, purchases may not be recorded because the purchase interval is longer than the survey period. We refer to this second case as infrequency of purchase (IFP). Thirdly, for some reason, commodity purchases may not be recorded, although the purchases do actually occur. Such misreporting can lead to the recording of zero expenditures. In this article, we only deal with the case of IFP.

Numerous articles have been devoted to solving the problem of zero expenditures generated by IFP: Deaton and Irish(1984), Kay et al. (1984), Keen (1986), Blundell and Meghir (1987), Pudney (1989, 1990), Griffiths and Valenzuela (1998), etc. Deaton and Irish(1984) present a $p$-tobit model that extends the tobit specification to model zero expenditures. Recorded data for expenditure on commodities is $1 / p$ times consumption during the survey period, where $p$ denotes the ratio of the survey period to the purchase period. This is applicable when goods are consumed during the survey period; however, expenditures are only observed with a probability $p$ because of infrequent purchasing (Deaton and Irish, 1984, p.63). Kay et al. (1984) extend the model proposed by Deaton and Irish (1984) by providing a stochastic relationship between expenditure and consumption in a sophisticated manner. Keen (1986) estimates a system of linear Engel functions that satisfy the adding-up condition and derives a consistent estimator based on the instrumental variables method. While the purchasing probabilities are constant parameters in Deaton and Irish (1984), Kay et al. (1984) and Keen (1986), Blundell and Meghir (1987) propose a model with probit-type purchasing probabilities. Griffiths and Valenzuela (1998) estimate a system of linear Engel functions and equivalence scales using a Bayesian method, and Pudney $(1989,1990)$ reviews numerous theoretical aspects associated with zero expenditures.

It is important to note the difference between expenditure and consumption when addressing the problem of zero expenditures. While expenditure is observable, consumption is not. Therefore, we can utilize data only for expenditures, which may include zero expenditures, and not for the true consumption of commodities. However, consumers derive utility from the consumption of commodities, not from expenditure. Thus, we have to introduce a stochastic relationship between expenditure and consumption in the model.

In this article, we propose a Bayesian approach for the estimation of a system of Engel functions using survey data that includes zero expenditures due to IFP. We explicitly introduce the stochastic relationship between expenditure and consumption in our Bayesian model. Using the Bayesian method, Hasegawa and Kozumi (2001) estimate a system of Working-Leser Engel functions that includes measurement errors. In this article, we employ the system used in Hasegawa and Kozumi (2001) and estimate the unobserved total consumption using the Bayesian framework. The posterior distributions of parameters play a central role in the Bayesian analysis; however, in complicated models, there exist

${ }^{1}$ See Keen (1986, p.277). 
cases where it is impossible to derive these distributions analytically. However, recent developments in the Markov chain Monte Carlo (MCMC) method permit us to sample unobservable parameters from their posterior distributions.

There are two advantages in our Bayesian approach. Firstly, although both models deal with the zero expenditure problem and satisfy the adding-up condition, the Bayesian approach enables us to estimate not only linear expenditure systems but also more flexible expenditure systems.

Secondly, we can estimate unobserved commodity consumption and total consumption. Thereafter, using unobserved total consumption, we can calculate inequality measures and total consumption elasticities. ${ }^{2}$ Since there exists a variation in the size of the households, the demographic aspects of households must be considered when measuring inequalities. Therefore, we introduce an equivalence scale in the estimation of Engel functions and calculate inequality measures based on the total consumption evaluated by this equivalence scale.

Estimation of demand and expenditure models using data with zero expenditures due to IFP are applied for varied purposes. Meghir and Robin (1992) use an infrequency of purchase model and estimate a demand system. Kimhi (1999) shows a model combining the double-hurdle and infrequency of purchase models to estimate household demand for tobacco. Newman et al. (2001) choose the double-hurdle and infrequency of purchase model for each meat product appropriately and estimate their expenditure equations. Madden (2000) estimates the expenditure elasticities of various items to calculate the poverty line. These constitute only a part of many empirical studies related to zero expenditures. This indicates that our Bayesian methods are applicable for diverse empirical analyses using data including them. However, it should be noted that zero expenditures on which we focus arise from IFP and not from corner solutions. Wales and Woodland (1983) propose the Kuhn-Tucker approach to estimate a demand system, and Fry et al. $(2000,2001)$ apply a compositional data analysis (CODA) for it, when the data include zero expenditures generated by corner solutions.

The article is organized as follows. In Section 2, we introduce a stochastic relationship between expenditure and consumption that deals with zero expenditures due to IFP and present a Bayesian model for estimating the system of Engel functions. In Section 3, we define the total consumption elasticity, the Gini coefficient and generalized entropy measures. In Section 4, we provide an empirical application of our approach to real data. In Section 5, we present concluding remarks and a few extensions of our approach.

\section{The Bayesian model}

\section{Expenditure and consumption}

Firstly, we introduce the following binary variables $D_{h i}$ :

$$
D_{h i}= \begin{cases}1 & \text { if household } h \text { purchases good } i \text { over the interview period } \\ 0 & \text { otherwise }\end{cases}
$$

\footnotetext{
${ }^{2}$ Hasegawa and Kozumi (2003) and Hasegawa et al. (2003) apply the MCMC method in order to estimate inequality measures.
} 
for $h=1, \cdots, H$ and $i=1, \cdots, M$, where $H$ is the number of households and $M$ is the number of goods. We assume that $D_{h i}$ are Bernoulli i.i.d. random variables with $\operatorname{Pr}\left(D_{h i}=1\right)=p_{i}$, where $p_{i}$ is the probability that a household will purchase good $i$ during the interview period. Next, we define $y_{h i}$ and $c_{h i}$ as observed expenditure and unobserved consumption for good $i$ and household $h$, respectively. Following Kay et al. (1984) and Keen (1986), we assume that

$$
y_{h i}=\left\{\begin{array}{ll}
\frac{1}{p_{i}}\left(c_{h i}+v_{h i}\right) & \text { if } D_{h i}=1 \\
0 & \text { if } D_{h i}=0
\end{array}, h=1, \cdots, H, i=1, \cdots, M,\right.
$$

where $v_{h i}$ are normally distributed i.i.d. disturbances, $v_{h i} \sim \mathrm{N}(0, \omega)$, and $D_{h i}$ and $v_{h i}$ are assumed to be independently distributed. Since we do not have observations for $y_{h i}$ when $D_{h i}=0$, we introduce the following latent variable $y_{h i}^{*}:^{3}$

$$
y_{h i}^{*}=\frac{1}{p_{i}}\left(c_{h i}+v_{h i}\right), \text { if } D_{h i}=0 .
$$

Using $y_{h i}$ and $y_{h i}^{*}$, we define $y_{h i}^{* *}$ as follows:

$$
y_{h i}^{* *}= \begin{cases}y_{h i} & \text { if } D_{h i}=1 \\ y_{h i}^{*} & \text { if } D_{h i}=0\end{cases}
$$

Equations (1), (2) and (3) are summarized as

$$
\left(\begin{array}{cccc}
p_{1} & & & \mathbf{O} \\
& \ddots & & \\
& & p_{m} & \\
\mathbf{O} & & & p_{M}
\end{array}\right)\left(\begin{array}{c}
y_{h 1}^{* *} \\
\vdots \\
y_{h m}^{* *} \\
y_{h M}^{* *}
\end{array}\right)=\left(\begin{array}{c}
c_{h 1} \\
\vdots \\
c_{h m} \\
c_{h M}
\end{array}\right)+\left(\begin{array}{c}
v_{h 1} \\
\vdots \\
v_{h m} \\
v_{h M}
\end{array}\right),
$$

where $m=M-1$. Or, more compactly,

$$
\left(\begin{array}{cc}
\boldsymbol{P}_{m} & \mathbf{0} \\
\mathbf{0}^{\prime} & p_{M}
\end{array}\right)\left(\begin{array}{c}
\boldsymbol{y}_{h}^{* *} \\
y_{h M}^{* *}
\end{array}\right)=\left(\begin{array}{c}
\boldsymbol{c}_{h} \\
c_{h M}
\end{array}\right)+\left(\begin{array}{c}
\boldsymbol{v}_{h} \\
v_{h M}
\end{array}\right)
$$

where

$$
\begin{aligned}
& \boldsymbol{P}_{m}=\operatorname{diag}\left(p_{1}, \cdots, p_{m}\right) \text { (diagonal matrix) } \\
& \boldsymbol{y}_{h}^{* *}=\left(\begin{array}{c}
y_{h 1}^{* *} \\
\vdots \\
y_{h m}^{* *}
\end{array}\right), \boldsymbol{c}_{h}=\left(\begin{array}{c}
c_{h 1} \\
\vdots \\
c_{h m}
\end{array}\right), \boldsymbol{v}_{h}=\left(\begin{array}{c}
v_{h 1} \\
\vdots \\
v_{h m}
\end{array}\right)
\end{aligned}
$$

From the adding-up condition, $x_{h}=\boldsymbol{\iota}_{m}^{\prime} \boldsymbol{c}_{h}+c_{h M}$ holds, where $x_{h}$ is the total consumption and $\iota_{m}=(\underbrace{1, \cdots, 1}_{m})^{\prime}$. Substituting the adding-up condition into (4), we have

$$
\left(\begin{array}{cc}
\boldsymbol{P}_{m} & \mathbf{0} \\
\mathbf{0}^{\prime} & p_{M}
\end{array}\right)\left(\begin{array}{c}
\boldsymbol{y}_{h}^{* *} \\
y_{h M}^{* *}
\end{array}\right)=\left(\begin{array}{cc}
\boldsymbol{I}_{m} & \mathbf{0} \\
-\boldsymbol{\iota}_{m}^{\prime} & 1
\end{array}\right)\left(\begin{array}{c}
\boldsymbol{c}_{h} \\
x_{h}
\end{array}\right)+\left(\begin{array}{c}
\boldsymbol{v}_{h} \\
v_{h M}
\end{array}\right), h=1, \cdots, H
$$

\footnotetext{
${ }^{3}$ See Griffiths and Valenzuela (1998).
} 
Since $v_{h i} \sim \mathrm{N}(0, \omega)$, we have the following joint distribution of $\boldsymbol{y}_{h}^{* *}, y_{h M}^{* *}$ in (5):

$$
p\left(\boldsymbol{y}_{h}^{* *}, y_{h M}^{* *} \mid \cdot\right) \propto\left(\prod_{i=1}^{M} p_{i}\right) \omega^{-M / 2} \exp \left[-\frac{1}{2 \omega}\left(\begin{array}{c}
\boldsymbol{v}_{h} \\
v_{h M}
\end{array}\right)^{\prime}\left(\begin{array}{c}
\boldsymbol{v}_{h} \\
v_{h M}
\end{array}\right)\right], h=1, \cdots, H .
$$

\section{Bayesian model for Engel curves}

We consider the following Working-Leser Engel curves: ${ }^{4}$

$$
\boldsymbol{w}_{h}=\boldsymbol{\alpha}+\boldsymbol{\beta} \log x_{h}+\boldsymbol{u}_{h}, \boldsymbol{u}_{h} \sim \mathrm{N}(\mathbf{0}, \boldsymbol{\Sigma}), h=1, \cdots, H,
$$

where $\boldsymbol{w}_{h}=\boldsymbol{c}_{h} / x_{h}$ is an $m \times 1$ share vector, $\boldsymbol{\alpha}=\left(\alpha_{1}, \cdots, \alpha_{m}\right)^{\prime}$ and $\boldsymbol{\beta}=$ $\left(\beta_{1}, \cdots, \beta_{m}\right)^{\prime}$ are parameter vectors, and $\boldsymbol{u}_{h}$ is an $m \times 1$ normally distributed error vector. Following Lancaster et al. (1999, p.459), we introduce an equivalence scale defined as

$$
m_{h}=n_{h}^{a}+\boldsymbol{\eta}^{\prime} \boldsymbol{z}_{h}
$$

where $n_{h}^{a}$ is the number of adults in household $h, \boldsymbol{\eta}$ is a $K \times 1$ scale parameter vector and $\boldsymbol{z}_{h}$ is a $K \times 1$ vector of demographic variables. ${ }^{5}$ Banks and Johnson (1994) indicate the importance of the relativity between the weight of children in a household and inequality. In this specification of the equivalence scale, it is simple to incorporate various demographic differences into the Engel curves. The results of their estimation for various age groups are referred to in 4.3.

Deflating the total consumption in (6) by the equivalence scale (7), we have

$$
\boldsymbol{w}_{h}=\boldsymbol{\alpha}+\boldsymbol{\beta} \log \frac{x_{h}}{m_{h}}+\boldsymbol{u}_{h}, \boldsymbol{u}_{h} \sim \mathrm{N}(\mathbf{0}, \boldsymbol{\Sigma}), h=1, \cdots, H .
$$

Defining $\boldsymbol{\Gamma}=(\boldsymbol{\alpha}, \boldsymbol{\beta})^{\prime}$ and $x_{h}^{*}=x_{h} / m_{h},(8)$ can be written as

$$
\boldsymbol{w}_{h}=\boldsymbol{\Gamma}^{\prime}\left(\begin{array}{c}
1 \\
\log x_{h}^{*}
\end{array}\right)+\boldsymbol{u}_{h}, h=1, \cdots, H
$$

For the Bayesian analysis, we consider the following prior information with regard to the hierarchical structure, ${ }^{6}$

$$
\left\{\begin{array}{l}
\log x_{h} \sim \mathrm{N}(\mu, \tau), \mu \sim \mathrm{N}\left(\mu_{*}, \kappa_{*}\right), \tau^{-1} \sim \operatorname{Gam}\left(a_{*}, b_{*}\right) \\
\boldsymbol{\gamma} \sim \mathrm{N}\left(\boldsymbol{\gamma}_{*}, \boldsymbol{G}_{*}\right), \boldsymbol{\Sigma}^{-1} \sim \mathrm{W}\left(\lambda_{*}, \boldsymbol{F}_{*}^{-1}\right), \omega^{-1} \sim \operatorname{Gam}\left(c_{*}, d_{*}\right) \\
p_{i} \sim \operatorname{Beta}\left(g_{*}, q_{*}\right), \boldsymbol{\eta} \sim \mathrm{N}\left(\boldsymbol{\eta}_{*}, \boldsymbol{A}_{*}\right),
\end{array}\right.
$$

where $\gamma=\operatorname{vec} \boldsymbol{\Gamma}, \operatorname{Gam}(a, b)$ denotes a gamma distribution with a shape parameter $a$ and scale parameter $b, \mathrm{~W}(a, \boldsymbol{A})$ denotes a Wishart distribution with degrees of freedom $a$ and a scale matrix $\boldsymbol{A}$ and $\operatorname{Beta}(a, b)$ denotes a beta distribution with parameters $a$ and $b$. The prior distribution of $\boldsymbol{c}_{h}$ can be derived

\footnotetext{
${ }^{4}$ See Working (1943) and Leser (1963). We delete a share equation from the system using the adding-up condition. That is $\sum_{i=1}^{M} \alpha_{i}=1, \sum_{i=1}^{M} \beta_{i}=0$.

${ }^{5}$ See Ray (1983), Lancaster and Ray (1998) and Lancaster et al. (1999) for details on this definition of equivalence scale.

${ }^{6}$ These prior distributions are often used in Bayesian analyses. See Hasegawa and Kozumi (2001).
} 
from the system of Engel functions (8). Further, we assume that the prior information of the latent variables $y_{h i}^{*}$ is noninformative. Given $(3),(5),(8)$ and (9), we can obtain the full conditional distributions (FCDs) of $\boldsymbol{\gamma}, \boldsymbol{\Sigma}^{-1}, \mu, \tau^{-1}$, $\omega^{-1}, \boldsymbol{c}_{h}(h=1, \cdots, H)$ and $y_{h i}^{*}(h=1, \cdots, H, i=1, \cdots, M)$. Using Gibbs sampling, we can easily sample these parameters from their FCDs. However, the closed forms of FCDs for other parameters cannot be derived because the FCDs of $x_{h}(h=1, \cdots, H), p_{i}(i=1, \cdots, M)$ and $\boldsymbol{\eta}$ are complicated functions. Therefore, we simulate these parameters using the Metropolis-Hastings (M-H) algorithm. The details of sampling algorithms are provided in the Appendix.

\section{Inequality measures and total consumption elasticities}

We use the expenditure data of households, including zero expenditures, for estimating the Engel functions. Expenditure is observable; however, in general, consumption is unobservable. As we mentioned in the introduction, one of the advantages of our Bayesian approach is that we can estimate unobserved consumption $c_{h i}$ and total consumption $x_{h}$ based on observable expenditure. The estimated posterior results provide information on the hidden true expenditure and consumption. Using these estimates enables us to calculate inequality measures and total consumption elasticities more accurately.

We use the Gini coefficient and the generalised entropy measure for measuring inequality. The Gini coefficient has played a central role in inequality literature and has many practical advantages (Cowell, 2000, pp.111-112). However, while the Gini coefficient does not satisfy population decomposability (Amiel and Cowell, 1999, p.138), the generalised entropy measure does. Therefore, we use the generalized entropy measure as well as the Gini coefficient.

The Gini coefficient and the generalised entropy measure are defined as follows:

$$
I_{\text {Gini }}=\frac{1}{2 H^{2} \bar{x}} \sum_{h=1}^{H} \sum_{k=1}^{H}\left|x_{h}-x_{k}\right|
$$

and

$$
I_{G E}(\theta)=\frac{1}{\theta^{2}-\theta}\left[\frac{1}{H} \sum_{h=1}^{H}\left(\frac{x_{h}}{\bar{x}}\right)^{\theta}-1\right],
$$

where $\theta$ is a real parameter. The generalised entropy measure is closely related to the Atkinson measure and two types of Theil measures, for $\theta=0,1$. We use the generalised entropy measure for cases of $\theta=-1,0,1,2$.

The total consumption elasticity of good $i$ is defined as follows:

$$
\left.\frac{\partial \log c_{i}}{\partial \log x}\right|_{c_{i}=\bar{c}_{i}, x=\bar{x}}=1+\frac{\beta_{i}}{\bar{w}_{i}}, \text { where } \bar{w}_{i}=\bar{c}_{i} / \bar{x} .
$$

The total consumption elasticities are calculated by using the sample means of $x$ and $c_{i}-\bar{x}$ and $\bar{c}_{i}$ - over the individual $x_{h}$ and $c_{h i}$.

\section{Application to real data}




\section{Data}

The data used for our empirical analysis are the micro-level survey data based on the Japanese Panel Survey of Consumers (JPSC) by the Institute for Research on Household Economics.

JPSC data provides details on consumption pertaining to young women in Japan. The survey began in 1993 and young women aged 24 to 34 years were the surveyed respondents. Later in 1997, the women aged 24 to 27 years were also included and the survey is continuing ever since.

In this article, we use Panel 8 of JPSC data, which is based on the survey in 2000. From the panel data we select, for the analysis, data pertaining to those households where there are no other adults except a husband and a wife. The number of households selected is 692. The women are aged between 27 and 41 years, and the average age is 34.1 years. The men are aged between 23 and 61 years, and the average age is 36.7 years.

JPSC data contains the expenditure data of households for the month of September. The average total expenditure of households in that month is 272,000 yen and the standard deviation is 121,000 yen. ${ }^{7}$

We divide the expense items of the data into ten items as follows: 'Food', 'Housing', 'Fuel, light and water charges', 'Furniture and household utensils', 'Clothes and footwear', 'Medical care', 'Transportation and communication', 'Education', 'Reading and recreation', and 'Miscellaneous'. Eight items are created from these ten items: Food, Housing, Fuel, Furniture, Clothing, Medical, Transport and Others. ${ }^{8}$

In the following empirical analysis, we set the dimension of demographic variable $\left(z_{h}\right) K=1$ in $(7)$, and use $z_{h}$ to denote the number of children. We determine the values of hyperparameters for simulation as follows:

$$
\begin{aligned}
& \gamma \sim \mathrm{N}\left(\mathbf{0}, 100 \times \boldsymbol{I}_{2 m}\right), \boldsymbol{\Sigma}^{-1} \sim \mathrm{W}\left(m+1,20 \times \boldsymbol{I}_{m}\right), \mu \sim \mathrm{N}(0,100) \\
& \tau^{-1} \sim \operatorname{Gam}(2,0.05), \omega^{-1} \sim \operatorname{Gam}(2,0.05), p_{i} \sim \operatorname{Beta}(1.5,1.5) \\
& \eta \sim \mathrm{N}(0,100)
\end{aligned}
$$

These hyperparameter values correspond to a less informative specification. The MCMC simulation was run for 30,000 iterations and the first 10,000 samples were discarded as a burn-in period. The posterior results obtained thereafter are generated using the Ox version 4.02 (Doornik, 2006).

\section{Posterior results}

The empirical results are summarized in Tables 1 to 4 . Several aspects of the results are described as follows.

Table 1 presents the number of zero expenditures in each item. The number of zeros in Housing, Furniture, Clothing and Medical are significantly higher

\footnotetext{
${ }^{7}$ The other descriptive statistics of the total expenditure of households are as follows. The median, the highest and the lowest expenditures are 250,000, 1,178,000, and 25,000 yen, respectively.

${ }^{8}$ Food='Food', Housing='Housing', Fuel='Fuel, light and water charges', Furniture='Furniture and household utensils', Clothing='Clothes and footwear', Medical='Medical care', Transport='Transportation and communication', and Others='Education'+'Reading and recreation'+'Miscellaneous'.
} 
than those in other items. The large number of zeros in Housing is a result of ownership of houses. Although the households that own their houses account for a certain ratio of the respondents, the expenditure on Housing in the data rules out imputed rent. The large number of zeros in Furniture, Clothing and Medical are due to IFS. There exist seven zeros in Food and eleven zeros in Others. It is unlikely that there exist households without any expenditure on food or the goods included in Others over a certain period; however, it has been observed in certain households. The reason why zeros are recorded in Food and Others is unclear. However, this may be possible due to the probability that certain respondents of the survey do not maintain their exact household accounts, because JPSC data are collected through a questionnaire.

Table 2 presents the posterior results for parameters. Calculating the ratios of the posterior mean to the posterior standard deviation (mean/sd) of $\alpha$ s and $\beta \mathrm{s}$, we find that their absolute values are higher than two, with the exception of a few parameters. $p_{i}$ 's values, which denote the probability of purchasing a commodity during the interview period, are lower in Housing, Furniture, Clothing and Medical than those in other items. Further, the order of sizes in the probabilities of Furniture, Housing, Medical and Clothing corresponds to that of the frequencies of zero expenditures in these items, as shown in Table 1. The posterior mean of $\eta$ is 0.3695 . The value of the equivalence scale for a reference household is 2.0 because the reference household comprises a husband and a wife with no children. Thus, we expect that $\eta$ lies in the interval $(0,1)$; indeed, we have $\hat{\eta}=0.3695 \in(0,1)$.

Table 3 presents the total consumption elasticities as calculated from the posterior results. This table demonstrates that Furniture, Transport and Others are luxury goods and that Food, Housing, Fuel, Clothing and Medical are necessary goods. The estimated elasticity of Clothing in our study is approximately one, and is lower than in many Japanese empirical studies where the elasticities exceed one. ${ }^{9}$ However, according to recent studies, the estimate is concluded to be proper. In fact, Ogawa and Okamura (2001) show that the elasticity of clothing is less than one and that it exhibits a declining trend after 1989 in Japan.

Table 4 presents the posterior results for inequality measures. The values in the columns 'raw', 'p.c.', 'e.s.' and 'mean' denote inequality measures based on total expenditure, per capita total expenditure, total expenditure deflated by the posterior equivalence scale and posterior mean of total consumption deflated by the equivalence scale, respectively. According to this table, the values for 'p.c.' are higher than those for 'raw' and 'e.s.' for each inequality measure. Furthermore, the values of 'e.s.' are higher than those of 'mean'. Thus, the inequality measures calculated using the posterior mean are lower than those using other data. It is known in general that the values of inequality measures based on data that includes measurement errors are higher than those for unobservable data that do not include them. ${ }^{10}$ This is consistent with our results and supports our Bayesian method for estimating inequality measures.

\section{Comparisons of posterior results}

\footnotetext{
${ }^{9}$ In Table 9, the estimate of the elasticity exceeds one when the number of goods and age groups increase. This aspect will be referred to in the next section.

${ }^{10}$ See Chakravarty and Eichhorn (1994), and Cowell (2000, p.137).
} 
In this section, we provide the results of two directions of sensitivity analyses. One of them deals with the case in which the number of goods $(M)$ changes. The other treats these cases where the number of demographic variables $(K)$ changes.

Table 5 presents the categories used in the estimated models with $M=5,6,7$ and 8 . This table also provides the number of zero expenditures of the items in the models. Table 6 presents the summary for the number of children in our data $(K=1,2$ and 3$) .{ }^{11}$ The case of $K=1$, described in the previous section, uses the age group of children from 0 to 18 years $\left(\eta_{1}\right)$ - the right column in Table 6 . We divide children into two age groups - children aged 0 to 12 years $\left(\eta_{1}\right)$ and those aged 13 to 18 years $\left(\eta_{2}\right)$-for the case of $K=2$ and into three age groups - children aged 0 to 6 years $\left(\eta_{1}\right), 7$ to 12 years $\left(\eta_{2}\right)$ and 13 to 18 years $\left(\eta_{3}\right)$ - for the case of $K=3$.

Table 7 presents the posterior means of the purchasing probabilities $p_{i}$. There are no substantial changes in the values of $p_{i}$ in the cases of $K=1,2$ and 3. The estimated probabilities are also similar to the actual relative frequencies denoted by 'data' in the table. Therefore, the posterior results for the purchasing probabilities $p_{i}$ are robust.

However, the equivalence scale is significantly influenced by the number of demographic variables and the number of categories in the model. Table 8 presents the posterior means of $\eta_{i}$ that are used in the equivalence scale defined in (7). The value of $\eta_{1}$ in the case of $K=1$ is greater than that in the cases of $K=2$ and $K=3$, except for the model with $M=7$; in addition, the value of $\eta_{1}$ in the case of $K=2$ is greater than that in the case of $K=3$. Further, the value of $\eta_{1}$ in the case of $K=2$ lies in the interval of $\left(\eta_{1}, \eta_{2}\right)$ in the case of $K=3$ for all $M$ in Table 8 . These findings for the values of $\eta_{i}$ are consistent with the definitions of age groups. However, in the models with $M=5,6$ and 7, the value of $\eta_{2}$ in the case of $K=2$ is greater than $\eta_{3}$ in the case of $K=3$. Both $\eta_{2}$ in the case of $K=2$ and $\eta_{3}$ in the case of $K=3$ denote the demographic parameters for the group of children aged 13 to 18 years in (7).

Table 9 provides the posterior means of the total consumption elasticities. The estimated elasticities of Food, Fuel, Furniture, Transportation and Others are almost the same, except for certain cases. The elasticity of Medical is greater than one, except for one case. The elasticities of Housing and Clothing tend to become large as $K$ and/or $M$ increase. As a result, Housing and Clothing become luxury goods in certain cases. Housing does not include imputed rent. Further, the elasticity of Housing would become small if imputed rents were included in the data. The ages of women in our data are between 27 and 41 years, as described above. It is highly probable that they have children belonging to the younger age groups in the case of $K=2$ or $K=3$, according to Table 6 . These households tend to be fashion conscious and highly concerned about their children's clothing. It is conceivable that their consumption of Clothing reacts to the movement of their total consumption. This would lead to the conclusion that the elasticity of Clothing is greater than one when $K=3$ for $M=7$ and $K=2$ and 3 for $M=8$. Similar reasons may hold true for these cases where the elasticities of Medical and Housing are

\footnotetext{
${ }^{11}$ We obtained the results that the posterior means of $\eta_{2}$ and/or $\eta_{3}$ in the equivalence scale are greater than one, when $K=2$ and $K=3$ in the models with $M=9$ and 10 . Since $\eta$ should lie in the interval $(0,1)$, we omit the posterior results of the models with $M=9$ and 10 .
} 
greater than one.

Table 10 presents the values of inequality measures. The values do not change substantially according to the number of demographic variables $(K)$ and the number of categories $(M)$. Therefore, the posterior means of inequality measures are robust with regard to $K$ and $M$.

\section{Conclusions and extensions}

Applying the Bayesian method, we estimated a system of Engel curves for Japanese households using JPSC micro data with zero expenditures and calculated inequality measures.

The Bayesian method enables us to estimate a model that overcomes the zero expenditure problem, satisfies the adding-up condition for a system of flexible Engel curves, introduces an equivalence scale and calculates inequality measures. As far as we know, few studies like ours have ever been attempted since it is not possible to estimate a model with the given conditions using non-Bayesian methods - this includes the maximum likelihood method.

We obtain the appropriate posterior results for the coefficients of a system of Engel functions and inequality measures. The sensitivity analyses - conducted by extending the categories of goods and demographic variables - indicate the robustness of our posterior results. The purchasing probabilities and inequality measures are particularly robust. The scale parameters of the equivalence scale result in appropriate values, although they are influenced by demographic variables. The consumption elasticities of goods are also valid depending on the categories of goods and demographic variables.

Our model can be extended as follows:

1. We can extend the Working-Leser Engel curves to a system of quadratic Engel curves.

2. By adding price variables to the model, we can estimate demand systems in order to use the advantages of panel data.

Firstly, instead of Equation (8), let us consider the following quadratic specification:

$$
\boldsymbol{w}_{h}=\boldsymbol{\alpha}+\boldsymbol{\beta} \log \frac{x_{h}}{m_{h}}+\boldsymbol{\delta}\left(\log \frac{x_{h}}{m_{h}}\right)^{2}+\boldsymbol{u}_{h}, \boldsymbol{u}_{h} \sim \mathrm{N}(\mathbf{0}, \boldsymbol{\Sigma}), h=1, \cdots, H,
$$

where $\boldsymbol{\delta}=\left(\delta_{1}, \cdots, \delta_{m}\right)^{\prime}$. For this modification, the Bayesian estimation procedure is similar as in Section 2 and Appendix. The main changes aim to redefine $\gamma$ as follows: ${ }^{12}$

$$
\boldsymbol{\gamma}=\operatorname{vec} \boldsymbol{\Gamma}, \text { such that } \boldsymbol{\Gamma}=(\boldsymbol{\alpha}, \boldsymbol{\beta}, \boldsymbol{\delta})^{\prime} .
$$

Next, we can extend the Working-Leser Engel curves (8) to the almost ideal demand system (Deaton and Muellbauer, 1980). The almost ideal demand

\footnotetext{
${ }^{12}$ See Hasegawa and Kozumi (2001).
} 
system, which satisfies the adding-up, homogeneity and symmetry conditions, can be written as follows:

$$
w_{i}=\alpha_{i}+\sum_{j=1}^{m} \psi_{i j} \log \left(\frac{\pi_{j}}{\pi_{M}}\right)+\beta_{i} \log \left(\frac{x^{*}}{a^{*}(\boldsymbol{\pi})}\right), i=1, \cdots, m,
$$

where $w_{i}$ is the budget share of good $i, \pi_{i}$ is the price of good $i, x$ is the total expenditure $x^{*}=x / \pi_{M}$ and

$$
\log a^{*}(\boldsymbol{\pi})=\alpha_{0}+\sum_{i=1}^{m} \alpha_{i} \log \left(\frac{\pi_{i}}{\pi_{M}}\right)+\frac{1}{2} \sum_{i=1}^{m} \sum_{j=1}^{m} \psi_{i j} \log \left(\frac{\pi_{i}}{\pi_{M}}\right) \log \left(\frac{\pi_{j}}{\pi_{M}}\right) .
$$

Using the linear algebra, (14) and (15) can be written as follows:

$$
\begin{aligned}
& \boldsymbol{w}=\boldsymbol{\alpha}+\left(\boldsymbol{\pi}^{\prime} \otimes \boldsymbol{I}_{m}\right) \boldsymbol{D} \boldsymbol{\psi}+\boldsymbol{\beta} \log \left(\frac{x^{*}}{a^{*}(\boldsymbol{\pi})}\right) \\
& \log a^{*}(\boldsymbol{\pi})=\alpha_{0}+\boldsymbol{\alpha}^{\prime} \boldsymbol{\pi}+\frac{1}{2}\left(\operatorname{vec} \boldsymbol{\pi} \boldsymbol{\pi}^{\prime}\right)^{\prime} \boldsymbol{D} \boldsymbol{\psi},
\end{aligned}
$$

where

$$
\boldsymbol{w}=\left(\begin{array}{c}
w_{1} \\
\vdots \\
w_{m}
\end{array}\right), \boldsymbol{\alpha}=\left(\begin{array}{c}
\alpha_{1} \\
\vdots \\
\alpha_{m}
\end{array}\right), \boldsymbol{\beta}=\left(\begin{array}{c}
\beta_{1} \\
\vdots \\
\beta_{m}
\end{array}\right), \boldsymbol{\pi}=\left(\begin{array}{c}
\log \left(\pi_{1} / \pi_{M}\right) \\
\vdots \\
\log \left(\pi_{m} / \pi_{M}\right)
\end{array}\right),
$$

$\boldsymbol{D}$ is a $m^{2} \times \frac{1}{2} m(m+1)$ duplication matrix, and $\boldsymbol{\psi}$ is a $\frac{1}{2} m(m+1) \times 1$ vector that is obtained from vec $\boldsymbol{\Psi}$ by eliminating all supradiagonal elements of $\boldsymbol{\Psi}=$ $\left(\begin{array}{ccc}\psi_{11} & \cdots & \psi_{1 m} \\ \vdots & \ddots & \vdots \\ \psi_{m 1} & \cdots & \psi_{m m}\end{array}\right) \cdot{ }^{13}$

By using the demand system (16) and (17), for household $h(h=1, \cdots, H)$ at period $t(t=1, \cdots, T)$, we define the following econometric model:

$$
\begin{aligned}
& \boldsymbol{w}_{h t}=\boldsymbol{\alpha}+\left(\boldsymbol{\pi}_{t}^{\prime} \otimes \boldsymbol{I}_{m}\right) \boldsymbol{D} \boldsymbol{\psi}+\boldsymbol{\beta} \log \left(\frac{x_{h t}^{*}}{a^{*}\left(\boldsymbol{\pi}_{t}\right)}\right)+\boldsymbol{u}_{h t}, \\
& \log a^{*}\left(\boldsymbol{\pi}_{t}\right)=\boldsymbol{\alpha}^{\prime} \boldsymbol{\pi}_{t}+\frac{1}{2}\left(\operatorname{vec} \boldsymbol{\pi}_{t} \boldsymbol{\pi}_{t}^{\prime}\right)^{\prime} \boldsymbol{D} \boldsymbol{}
\end{aligned}
$$

where $\boldsymbol{w}_{h t}$ is an $m \times 1$ share vector and $\boldsymbol{\pi}_{t}=\left(\log \left(\pi_{t 1} / \pi_{t M}\right), \cdots, \log \left(\pi_{t m} / \pi_{t M}\right)\right)^{\prime}$, $x_{h t}^{*}=x_{h t} /\left(m_{h t} \pi_{t M}\right), m_{h t}$ is an equivalence scale and $\boldsymbol{u}_{h t}$ is a vector of normally distributed disturbances. ${ }^{14}$ We can introduce the similar structure for household's expenditure on good $i$ at period $t$, say $y_{h t i}^{* *}(h=1, \cdots, H, t=$ $1, \cdots, T, i=1, \cdots, M)$, as described in Section 2. Combining the structure for $y_{h t i}^{* *}$ with the demand system (18) and (19) and noting that the demand system (18) and (19) are linear in coefficient parameters $(\boldsymbol{\alpha}, \boldsymbol{\beta}, \boldsymbol{\psi})$ in terms of their FCDs, we can apply the method in this article to the estimation of the demand system with zero expenditures.

\footnotetext{
${ }^{13}$ For the details of duplication matrix, see Magnus and Neudecker (1999, pp.48-49).

${ }^{14}$ In (19), we have deleted $\alpha_{0}$.
} 
Furthermore, from these two extensions, our Bayesian approach can be applied to the quadratic almost ideal demand system (Banks et al., 1997) as well as the almost ideal demand system.

\section{References}

Amiel, Y. and Cowell, F. A. (1999). Thinking about Inequality, Cambridge University Press: Cambridge.

Banks, J. and Johnson, P. (1994). 'Equivalence scale relativities revisited,' Economic Journal, Vol.104, 883-890.

Banks, J., Blundell, R. and Lewbel, A. (1997). 'Quadratic Engel curves and consumer demand,' Review of Economics and Statistics, Vol.79, 527-539.

Blundell, R. and Meghir, C. (1987). 'Bivariate alternatives to the Tobit model,' Journal of Econometrics, Vol.34, 179-200.

Chakravarty, S. R. and Eichhorn, W. (1994). 'Measurement of income inequality: Observed versus true data,' in W. Eichhorn (ed.), Models and Measurement of Welfare and Inequality, Springer-Verlag: Berlin, 28-32.

Chib, S. and Greenberg, E. (1995). 'Understanding the Metropolis-Hastings algorithm,' American Statistician, Vol.49, 327-335.

Chib, S., Greenberg, E. and Winkelmann, R. (1998). 'Posterior simulation and Bayes factors in panel count data models,' Journal of Econometrics, Vol.86, 33-54.

Cowell, F. A. (2000). 'Measurement of inequality'' in A. B. Atkinson and F. Bourguignon (eds), Handbook of Income Distribution, North-Holland: Amsterdam, 87-166.

Deaton, A . and Irish, M. (1984). 'Statistical models for zero expenditures in household budgets,' Journal of Public Economics, Vol.23, 59-80.

Deaton, A. and Muellbauer, J. (1980). 'An almost ideal demand system,' American Economic Review, Vol.70, 312-326.

Doornik, J.A. (2006). An Object-oriented Matrix Programming Language: $\mathrm{Ox}^{T M} 4$, Timberlake Consultants Ltd: London.

Fry, J. M., Fry, T. R. L. and McLaren, K. R. (2000). 'Compositional data analysis and zeros in micro data,' Applied Economics Vol.32, 953-959.

Fry, J. M., Fry, T. R. L., McLaren, K. R. and Smith, T. N. (2001). 'Modelling zeros in microdata,' Applied Economics Vol.33, 383-392.

Griffiths, W. and Valenzuela, M.R. (1998). 'Missing data from infrequency of purchase: Bayesian estimation of a linear expenditure system,' in T. B. Fomby, and R. C. Hill (eds), Advances in Econometrics: Messy Data - Missing Observations, Outliers, and Mixed-Frequency Data Vol.13, JAI Press: Greenwich, 75-102. 
Hasegawa, H. and Kozumi, H. (2001). 'Bayesian analysis on Engel curves estimation with measurement errors and an instrumental variable,' Journal of Business \& Economic Statistics, Vol.19, 292-298.

Hasegawa, H. and Kozumi, H. (2003). "Estimation of Lorenz curves: A Bayesian nonparametric approach," Journal of Econometrics, vol.115, 277291.

Hasegawa, H., Van Hoa, T. and Valenzuela, M. R. (2003). 'HOGLEX demand systems and welfare policy in major ASEAN developing countries: A Bayesian analysis using expenditure unit records,' Journal of Quantitative Economics, Vol.1, 52-65.

Kay, J. A., Keen, M. J. and Morris, C. N. (1984). 'Estimating consumption from expenditure data,' Journal of Public Economics, Vol.23, 169-181.

Keen, M. (1986). 'Zero expenditures and the estimation of Engel curves,' Journal of Applied Econometrics, Vol.1, 277-286.

Kimhi, A. (1999). 'Double-hurdle and purchase-infrequency demand analysis: A feasible integrated approach,' European Review of Agricultural Economics, Vol.26, 425-442.

Lancaster, G. and Ray, R. (1998). 'Comparison of alternative models of household equivalence scales: The Austrian evidence on unit record data,' Economic Record, Vol.74, 1-14.

Lancaster, G., Ray, R. and Valenzuela, M. R. (1999). 'A cross-country study of household poverty and inequality on unit record household budget data,' Economic Development and Cultural Change, Vol.48, 177-208.

Leser, C.E.V. (1963). 'Forms of Engel functions,' Econometrica, Vol.31, 694703.

Lütkepohl, H. (1996). Handbook of Matrices, Wiley: Chichester.

Madden, D. (2000). 'Relative or absolute poverty lines: A new approach,' Review of Income and Wealth, Vol.46, 181-199.

Magnus, J. R. and Neudecker, H. (1999). Matrix Differential Calculus with Applications in Statistics and Econometrics, Revised ed.,Wiley: Chichester.

Meghir, C. and Robin, J-M. (1992). 'Frequency of purchase and the estimation of demand systems,' Journal of Econometrics, Vol.53, 53-85.

Newman, C., Henchion, M. and Matthews, A. (2001). 'Infrequency of purchase and double-hurdle models of Irish households' meat expenditure,' European Review of Agricultural Economics, Vol.28, 393-412.

Ogawa, K. and Okamura, K. (2001). 'Leisure and Consumption Demand,' (in Japanese,) Economic Review (Keizai Kenkyu), Vol.52, 132-142.

Pudney, S. (1989). Modelling Individual Choice: The Econometrics of Corners, Kinks and Holes, Basil Blackwell: Oxford. 
Pudney, S. (1990). 'The estimation of Engel curves,' (with discussion), in G. D. Myles (ed.), Measurement and Modelling in Economics, North-Holland: Amsterdam, 267-323.

Ray, R. (1983). 'Measuring the costs of children: An alternative approach,' Journal of Public Economics, Vol.22, 89-102.

Tierney, L. (1994). 'Markov chains for exploring posterior distributions,' (with discussion), Annals of Statistics, Vol.22, 1701-1762.

Wales, T. J. and Woodland, A. D. (1983). 'Estimation of consumer demand systems with binding non-negativity constraints,' Journal of Econometrics, Vol.21, 263-285.

Working, H. (1943). 'Statistical laws of family expenditure,' Journal of the American Statistical Association, vol.38, 43-56.

\section{Appendix A. Sampling algorithms}

\section{A.1. Full conditional distributions of parameters}

The FCDs of $\boldsymbol{\gamma}, \boldsymbol{\Sigma}^{-1}, \mu, \tau^{-1}, \omega^{-1}, \boldsymbol{c}_{h}(h=1, \cdots, H)$ and $y_{h i}^{*}(h=1, \cdots, H$, $i=1, \cdots, M)$ are as follows:

- FCD of $\gamma$ :

$$
\begin{aligned}
\gamma \mid \cdots \sim \mathrm{N}\left(\boldsymbol{\gamma}_{* *}, \boldsymbol{G}_{* *}\right) \\
\boldsymbol{G}_{* *}=\left[\boldsymbol{G}_{*}^{-1}+\left(\boldsymbol{\Sigma}^{-1} \otimes \boldsymbol{X}_{*}^{\prime} \boldsymbol{X}_{*}\right)\right]^{-1} \\
\boldsymbol{\gamma}_{* *}=\boldsymbol{G}_{* *}\left[\boldsymbol{G}_{*}^{-1} \boldsymbol{\gamma}_{*}+\left(\boldsymbol{\Sigma}^{-1} \otimes \boldsymbol{I}_{2}\right) \operatorname{vec}\left(\boldsymbol{X}_{*}^{\prime} \boldsymbol{W}\right)\right],
\end{aligned}
$$

where ' $\mid \cdots$ ' denotes conditioning on the values of all other parameters and data,

$$
\boldsymbol{X}=\left(\begin{array}{cc}
1 & \log x_{1}^{*} \\
\vdots & \vdots \\
1 & \log x_{H}^{*}
\end{array}\right), \boldsymbol{W}=\left(\begin{array}{c}
\boldsymbol{w}_{1}^{\prime} \\
\vdots \\
\boldsymbol{w}_{H}^{\prime}
\end{array}\right)
$$

and $\otimes$ denotes a Kronecker product.

- FCD of $\Sigma^{-1}$ :

$$
\begin{aligned}
\boldsymbol{\Sigma}^{-1} \mid \cdots & \sim \mathrm{W}\left(\lambda_{* *}, \boldsymbol{F}_{* *}^{-1}\right) \\
\lambda_{* *} & =\lambda_{*}+H, \boldsymbol{F}_{* *}=\boldsymbol{F}_{*}+\sum_{h=1}^{H} \boldsymbol{u}_{h} \boldsymbol{u}_{h}^{\prime} .
\end{aligned}
$$

- FCD of $\mu$ :

$$
\begin{aligned}
\mu \mid \cdots & \sim \mathrm{N}\left(\mu_{* *}, \kappa_{* *}\right) \\
\kappa_{* *} & =\left(\frac{1}{\kappa_{*}}+\frac{H}{\tau}\right)^{-1}, \mu_{* *}=\kappa_{* *}\left(\frac{\mu_{*}}{\kappa_{*}}+\frac{1}{\tau} \sum_{h=1}^{H} \log x_{h}\right) .
\end{aligned}
$$


- FCD of $\tau^{-1}$ :

$$
\begin{aligned}
\tau^{-1} \mid \cdots & \sim \operatorname{Gam}\left(a_{* *}, b_{* *}\right) \\
a_{* *} & =a_{*}+\frac{H}{2}, b_{* *}=b_{*}+\frac{1}{2} \sum_{h=1}^{H}\left(\log x_{h}-\mu\right)^{2} .
\end{aligned}
$$

- FCD of $\omega^{-1}$ :

$$
\begin{aligned}
\omega^{-1} \mid \cdots & \sim \operatorname{Gam}\left(c_{* *}, d_{* *}\right), i=1, \cdots, M \\
c_{* *} & =c_{*}+\frac{M H}{2}, d_{* *}=d_{*}+\frac{1}{2} \sum_{h=1}^{H}\left(\begin{array}{c}
\boldsymbol{v}_{h} \\
v_{h M}
\end{array}\right)^{\prime}\left(\begin{array}{c}
\boldsymbol{v}_{h} \\
v_{h M}
\end{array}\right) .
\end{aligned}
$$

- FCD of $c_{h}$ :

$$
\begin{aligned}
\boldsymbol{c}_{h} \mid \cdots \sim \mathrm{N}\left(\boldsymbol{c}_{h * *}, \boldsymbol{\Sigma}_{h * *}\right), h=1, \cdots, H \\
\boldsymbol{\Sigma}_{h * *}=\left[\frac{1}{x_{h}^{2}} \boldsymbol{\Sigma}^{-1}+\frac{1}{\omega}\left(\boldsymbol{I}_{m}+\boldsymbol{\iota}_{m} \boldsymbol{\iota}_{m}^{\prime}\right)\right]^{-1} \\
\boldsymbol{c}_{h * *}=\boldsymbol{\Sigma}_{h * *}\left[\frac{1}{x_{h}} \boldsymbol{\Sigma}^{-1}\left(\boldsymbol{\alpha}+\boldsymbol{\beta} \log x_{h}^{*}\right)+\frac{1}{\omega}\left\{\left(x_{h}-p_{M} y_{h M}^{* *}\right) \boldsymbol{\iota}_{m}+\boldsymbol{P}_{m} \boldsymbol{y}_{h}^{* *}\right\}\right] .
\end{aligned}
$$

- FCD of $\boldsymbol{y}_{h i}^{*}:$ When $D_{h i}=0$,

$$
y_{h i}^{*} \mid \cdots \sim \mathrm{N}\left(\frac{c_{h i}}{p_{i}}, \frac{\omega}{p_{i}^{2}}\right), h=1, \cdots, H, i=1, \cdots, M .
$$

Using Gibbs sampling, we can easily simulate the above-mentioned parameters from their FCDs.

However, since the FCDs of $x_{h}(h=1, \cdots, H), p_{i}(i=1, \cdots, M)$ and $\boldsymbol{\eta}$ are complicated functions, the closed forms of FCDs for the parameters cannot be derived. Therefore, we simulate these parameters using the Metropolis-Hasting (M-H) algorithm. ${ }^{15}$

\section{A.2. Sampling of $\boldsymbol{x}_{h}$}

Let $f(x)$ and $q\left(x^{\prime}, x\right)$ denote the target and proposal densities of a transition from $x^{\prime}$ to $x$, respectively. The Metropolis-Hastings (M-H) algorithm can be described as follows:

1. At the $(t+1)$ th iteration, given the current sample $x^{(t)}$, sample $x$ from the proposal density $q\left(x^{(t)}, x\right)$.

2. Generate $u \sim \mathrm{U}(0,1)$, a uniform distribution on $(0,1)$ and take

$$
x^{(t+1)}= \begin{cases}x & \text { if } u<\min \left\{\frac{f(x) q\left(x, x^{(t)}\right)}{f\left(x^{(t)}\right) q\left(x^{(t)}, x\right)}, 1\right\} \\ x^{(t)} & \text { otherwise. }\end{cases}
$$

\footnotetext{
${ }^{15}$ See, for example, Tierney (1994) and Chib and Greenberg (1995).
} 
There are several suggestions for choosing the proposal density. In this article, we employ a tailored proposal density. ${ }^{16}$

Since the FCD of $x_{h}$ is

$$
\begin{gathered}
p\left(x_{h} \mid \cdots\right) \propto \frac{1}{x_{h}} \exp \left[-\frac{1}{2 \tau}\left(\log x_{h}-\mu\right)^{2}\right]\left(\frac{1}{x_{h}}\right)^{m} \exp \left(-\frac{1}{2} \boldsymbol{u}_{h}^{\prime} \boldsymbol{\Sigma}^{-1} \boldsymbol{u}_{h}\right) \exp \left(-\frac{1}{2 \omega} v_{h M}^{2}\right) \\
=\left(\frac{1}{x_{h}}\right)^{m+1} \exp \left[-\frac{1}{2}\left\{\boldsymbol{u}_{h}^{\prime} \boldsymbol{\Sigma}^{-1} \boldsymbol{u}_{h}+\frac{1}{\omega} v_{h M}^{2}+\frac{1}{\tau}\left(\log x_{h}-\mu\right)^{2}\right\}\right],
\end{gathered}
$$

we have

$$
\log p\left(x_{h} \mid \cdots\right)=\text { const. }-(m+1) \log x_{h}-\frac{1}{2}\left[\boldsymbol{u}_{h}^{\prime} \boldsymbol{\Sigma}^{-1} \boldsymbol{u}_{h}+\frac{1}{\omega} v_{h M}^{2}+\frac{1}{\tau}\left(\log x_{h}-\mu\right)^{2}\right] .
$$

The first and second derivatives of $\log p\left(x_{h} \mid \cdots\right)$ with respect to $x_{h}$ are as follows: ${ }^{17}$

$$
\begin{aligned}
& \frac{\partial \log p\left(x_{h} \mid \cdots\right)}{\partial x_{h}}=-\frac{m+1}{x_{h}}-\frac{x_{h}-\left(p_{M} y_{h M}^{* *}+\boldsymbol{\iota}_{m}^{\prime} \boldsymbol{c}_{h}\right)}{\omega}-\frac{\log x_{h}-\mu}{\tau x_{h}}-\frac{1}{2} \frac{\partial \boldsymbol{u}_{h}^{\prime} \boldsymbol{\Sigma}^{-1} \boldsymbol{u}_{h}}{\partial x_{h}} \\
& \frac{\partial^{2} \log p\left(x_{h} \mid \cdots\right)}{\partial x_{h}^{2}}=\frac{1}{x_{h}^{2}}\left[m+1+\frac{1}{\tau}\left(\log x_{h}-\mu-1\right)\right]-\frac{1}{\omega}-\frac{1}{2} \frac{\partial^{2} \boldsymbol{u}_{h}^{\prime} \boldsymbol{\Sigma}^{-1} \boldsymbol{u}_{h}}{\partial x_{h}^{2}}
\end{aligned}
$$

where

$$
\begin{aligned}
& \frac{\partial \boldsymbol{u}_{h}^{\prime} \boldsymbol{\Sigma}^{-1} \boldsymbol{u}_{h}}{\partial x_{h}}=2\left(\frac{\partial \boldsymbol{u}_{h}}{\partial x_{h}}\right)^{\prime} \boldsymbol{\Sigma}^{-1} \boldsymbol{u}_{h} \\
& \frac{\partial^{2} \boldsymbol{u}_{h}^{\prime} \boldsymbol{\Sigma}^{-1} \boldsymbol{u}_{h}}{\partial x_{h}^{2}}=2\left(\frac{\partial \boldsymbol{u}_{h}}{\partial x_{h}}\right)^{\prime} \boldsymbol{\Sigma}^{-1}\left(\frac{\partial \boldsymbol{u}_{h}}{\partial x_{h}}\right)+2 \boldsymbol{u}_{h}^{\prime} \boldsymbol{\Sigma}^{-1}\left(\frac{\partial^{2} \boldsymbol{u}_{h}}{\partial x_{h}^{2}}\right) \\
& \frac{\partial \boldsymbol{u}_{h}}{\partial x_{h}}=-\frac{1}{x_{h}}\left(\boldsymbol{w}_{h}+\boldsymbol{\beta}\right) \\
& \frac{\partial^{2} \boldsymbol{u}_{h}}{\partial x_{h}^{2}}=\frac{1}{x_{h}^{2}}\left(2 \boldsymbol{w}_{h}+\boldsymbol{\beta}\right) .
\end{aligned}
$$

Then, employing the first and the second derivatives of $\log p\left(x_{h} \mid \cdots\right)$, we can obtain the mode of $\log p\left(x_{h} \mid \cdots\right)$, say $\hat{x}_{h}$. Now, define the tailored proposal density as follows:

$$
q\left(x_{h}^{\prime}, x_{h}\right)=q\left(x_{h}\right)=f_{t}\left(x_{h} \mid \hat{x}_{h}, s_{h}^{2}, \nu\right) \propto\left[1+\frac{1}{\nu s_{h}^{2}}\left(x_{h}-\hat{x}_{h}\right)^{2}\right]^{-(\nu+1) / 2},
$$

where $\nu$ is an adjustable constant, ${ }^{18} f_{t}(\cdot \mid a, b, \nu)$ denotes a $t$ density with $\nu$ degrees of freedom, location parameter $a$ and scale parameter $b$, and

$$
s_{h}^{2}=\left[-\left.\frac{\partial^{2} \log p\left(x_{h} \mid \cdots\right)}{\partial x_{h}^{2}}\right|_{x_{h}=\hat{x}_{h}}\right]^{-1}
$$

provided $s_{h}^{2}>0$. Thus, we have the following M-H algorithm with a tailored proposal density:

\footnotetext{
${ }^{16}$ See, for example, Chib et al. (1998).

${ }^{17}$ For the derivatives of vectors and matrices, see Lütkepohl (p.175, 1996).

${ }^{18}$ In Section 4 , we set $\nu=7$.
} 
1. At the $(t+1)$ th iteration, given the current sample $x_{h}^{(t)}$, sample $x_{h}$ from the proposal density $q\left(x_{h}\right)$ until $x_{h} \geq \sum_{i=1}^{m} c_{h i}$.

2. Generate $u \sim \mathrm{U}(0,1)$, the uniform distribution on $(0,1)$ and take

$$
x_{h}^{(t+1)}= \begin{cases}x_{h} & \text { if } u<\min \left\{\frac{\mathrm{e}^{\log p\left(x_{h} \mid \cdots\right)} q\left(x_{h}^{(t)}\right)}{\mathrm{e}^{\log p\left(x_{h}^{(t)} \mid \cdots\right)} q\left(x_{h}\right)}, 1\right\} \\ x_{h}^{(t)} & \text { otherwise. }\end{cases}
$$

\section{A.3. Sampling of $p$}

Since

$$
\begin{aligned}
& p(\boldsymbol{p} \mid \cdots) \propto \prod_{i=1}^{M} p_{i}^{g_{*}-1}\left(1-p_{i}\right)^{q_{*}-1} p_{i}^{H+\sum_{h=1}^{H} D_{h i}}\left(1-p_{i}\right)^{H-\sum_{h=1}^{H} D_{h i}} \\
& \quad \times \exp \left[-\frac{1}{2 \omega} \sum_{h=1}^{H}\left(p_{i} y_{h i}^{* *}-c_{h i}\right)^{2}\right] \\
& =\prod_{i=1}^{M} p_{i}^{g_{*}+H+\sum_{h=1}^{H} D_{h i}-1}\left(1-p_{i}\right)^{q_{*}+H-\sum_{h=1}^{H} D_{h i}-1} \exp \left[-\frac{1}{2 \omega} \sum_{h=1}^{H}\left(p_{i} y_{h i}^{* *}-c_{h i}\right)^{2}\right],
\end{aligned}
$$

we have

$$
\begin{aligned}
& \log p(\boldsymbol{p} \mid \cdots)=\text { const. }+\sum_{i=1}^{M}\left(g_{*}+H+\sum_{h=1}^{H} D_{h i}-1\right) \log p_{i} \\
& +\sum_{i=1}^{M}\left(q_{*}+H-\sum_{h=1}^{H} D_{h i}-1\right) \log \left(1-p_{i}\right)-\frac{1}{2 \omega} \sum_{i=1}^{M} \sum_{h=1}^{H}\left(p_{i} y_{h i}^{* *}-c_{h i}\right)^{2} .
\end{aligned}
$$

The first and second derivatives of $\log p(\boldsymbol{p} \mid \cdots)$ with respect to $p_{i}$ are as follows:

$$
\begin{aligned}
& \frac{\partial \log p(\boldsymbol{p} \mid \cdots)}{\partial p_{i}}=\frac{1}{p_{i}}\left(g_{*}+H+\sum_{h=1}^{H} D_{h i}-1\right) \\
& -\frac{1}{1-p_{i}}\left(q_{*}+H-\sum_{h=1}^{H} D_{h i}-1\right)-\frac{1}{\omega} \sum_{h=1}^{H}\left(p_{i} y_{h i}^{* *}-c_{h i}\right) y_{h i}^{* *} \\
& \frac{\partial^{2} \log p(\boldsymbol{p} \mid \cdots)}{\partial p_{i}^{2}}=-\frac{1}{p_{i}^{2}}\left(g_{*}+H+\sum_{h=1}^{H} D_{h i}-1\right) \\
& -\frac{1}{\left(1-p_{i}\right)^{2}}\left(q_{*}+H-\sum_{h=1}^{H} D_{h i}-1\right)-\frac{1}{\omega} \sum_{h=1}^{H} y_{h i}^{* * 2} .
\end{aligned}
$$

Then, using the first and the second derivatives of $\log p(\boldsymbol{p} \mid \cdots)$, we can obtain the mode of $\log p(\boldsymbol{p} \mid \cdots)$, say $\hat{\boldsymbol{p}}$. Now, define the tailored proposal density as follows:

$$
q\left(\boldsymbol{p}^{\prime}, \boldsymbol{p}\right)=q(\boldsymbol{p})=f_{M t}(\boldsymbol{p} \mid \hat{\boldsymbol{p}}, \boldsymbol{S}, \nu) \propto\left[1+\frac{1}{\nu}(\boldsymbol{p}-\hat{\boldsymbol{p}})^{\prime} \boldsymbol{S}^{-1}(\boldsymbol{p}-\hat{\boldsymbol{p}})\right]^{-(\nu+M) / 2},
$$


where $\nu$ is an adjustable constant, ${ }^{19}$ and $f_{M t}(\cdot \mid \boldsymbol{a}, \boldsymbol{A}, \nu)$ denotes a multivariate $t$ density with $\nu$ degrees of freedom, location parameter vector $\boldsymbol{a}$ and scale parameter matrix $\boldsymbol{A}$, and

$$
\boldsymbol{S}=\left[-\left.\frac{\partial^{2} \log p(\boldsymbol{p} \mid \cdots)}{\partial \boldsymbol{p} \partial \boldsymbol{p}^{\prime}}\right|_{\boldsymbol{p}=\hat{\boldsymbol{p}}}\right]^{-1},
$$

provided $\boldsymbol{S}$ is positive definite. Thus, we have the following M-H algorithm with a tailored proposal density:

1. At the $(t+1)$ th iteration, given the current sample $\boldsymbol{p}^{(t)}$, sample $\boldsymbol{p}$ from the proposal density $q(\boldsymbol{p})$.

2. Generate $u \sim \mathrm{U}(0,1)$, the uniform distribution on $(0,1)$ and take

$$
\boldsymbol{p}^{(t+1)}= \begin{cases}\boldsymbol{p} & \text { if } u<\min \left\{\frac{\mathrm{e}^{\log p(\boldsymbol{p} \mid \cdots)} q\left(\boldsymbol{p}^{(t)}\right)}{\mathrm{e}^{\log p\left(\boldsymbol{p}^{(t)} \mid \cdots\right)} q(\boldsymbol{p})}, 1\right\} \\ \boldsymbol{p}^{(t)} & \text { otherwise. }\end{cases}
$$

\section{A.4. Sampling of $\eta$}

Since

$$
\begin{gathered}
p(\boldsymbol{\eta} \mid \cdots) \propto \exp \left[-\frac{1}{2}\left(\boldsymbol{\eta}-\boldsymbol{\eta}_{*}\right)^{\prime} \boldsymbol{A}_{*}^{-1}\left(\boldsymbol{\eta}-\boldsymbol{\eta}_{*}\right)\right] \exp \left(-\frac{1}{2} \sum_{h=1}^{H} \boldsymbol{u}_{h}^{\prime} \boldsymbol{\Sigma}^{-1} \boldsymbol{u}_{h}\right) \\
\quad=\exp \left[-\frac{1}{2}\left\{\left(\boldsymbol{\eta}-\boldsymbol{\eta}_{*}\right)^{\prime} \boldsymbol{A}_{*}^{-1}\left(\boldsymbol{\eta}-\boldsymbol{\eta}_{*}\right)+\sum_{h=1}^{H} \boldsymbol{u}_{h}^{\prime} \boldsymbol{\Sigma}^{-1} \boldsymbol{u}_{h}\right\}\right]
\end{gathered}
$$

we have

$$
\log p(\boldsymbol{\eta} \mid \cdots)=\text { const. }-\frac{1}{2}\left[\left(\boldsymbol{\eta}-\boldsymbol{\eta}_{*}\right)^{\prime} \boldsymbol{A}_{*}^{-1}\left(\boldsymbol{\eta}-\boldsymbol{\eta}_{*}\right)+\sum_{h=1}^{H} \boldsymbol{u}_{h}^{\prime} \boldsymbol{\Sigma}^{-1} \boldsymbol{u}_{h}\right]
$$

The first and second derivatives of $\log p(\boldsymbol{\eta} \mid \cdots)$ by $\boldsymbol{\eta}$ are as follows: ${ }^{20}$

$$
\begin{aligned}
& \frac{\partial \log p(\boldsymbol{\eta} \mid \cdots)}{\partial \boldsymbol{\eta}}=-\boldsymbol{A}_{*}^{-1}\left(\boldsymbol{\eta}-\boldsymbol{\eta}_{*}\right)-\frac{1}{2} \sum_{h=1}^{H} \frac{\partial \boldsymbol{u}_{h}^{\prime} \boldsymbol{\Sigma}^{-1} \boldsymbol{u}_{h}}{\partial \boldsymbol{\eta}} \\
& \frac{\partial^{2} \log p(\boldsymbol{\eta} \mid \cdots)}{\partial \boldsymbol{\eta} \partial \boldsymbol{\eta}^{\prime}}=-\boldsymbol{A}_{*}^{-1}-\frac{1}{2} \sum_{h=1}^{H} \frac{\partial^{2} \boldsymbol{u}_{h}^{\prime} \boldsymbol{\Sigma}^{-1} \boldsymbol{u}_{h}}{\partial \boldsymbol{\eta} \partial \boldsymbol{\eta}^{\prime}},
\end{aligned}
$$

\footnotetext{
${ }^{19}$ In Section 4 , we set $\nu=7$

${ }^{20}$ See Lütkepohl (p.175, 1996).
} 
where

$$
\begin{aligned}
& \frac{\partial \boldsymbol{u}_{h}^{\prime} \boldsymbol{\Sigma}^{-1} \boldsymbol{u}_{h}}{\partial \boldsymbol{\eta}}=2\left(\frac{\partial \boldsymbol{u}_{h}}{\partial \boldsymbol{\eta}^{\prime}}\right)^{\prime} \boldsymbol{\Sigma}^{-1} \boldsymbol{u}_{h} \\
& \frac{\partial^{2} \boldsymbol{u}_{h}^{\prime} \boldsymbol{\Sigma}^{-1} \boldsymbol{u}_{h}}{\partial \boldsymbol{\eta} \partial \boldsymbol{\eta}^{\prime}}=2\left(\frac{\partial \boldsymbol{u}_{h}}{\partial \boldsymbol{\eta}^{\prime}}\right)^{\prime} \boldsymbol{\Sigma}^{-1} \frac{\partial \boldsymbol{u}_{h}}{\partial \boldsymbol{\eta}^{\prime}}+2\left(\boldsymbol{u}_{h}^{\prime} \boldsymbol{\Sigma}^{-1} \otimes \boldsymbol{I}_{K}\right) \frac{\partial}{\partial \boldsymbol{\eta}^{\prime}}\left[\operatorname{vec}\left(\frac{\partial \boldsymbol{u}_{h}^{\prime}}{\partial \boldsymbol{\eta}}\right)\right] \\
& \frac{\partial \boldsymbol{u}_{h}}{\partial \boldsymbol{\eta}^{\prime}}=\frac{\boldsymbol{\beta} \boldsymbol{z}_{h}^{\prime}}{m_{h}} \\
& \frac{\partial}{\partial \boldsymbol{\eta}^{\prime}}\left[\operatorname{vec}\left(\frac{\partial \boldsymbol{u}_{h}^{\prime}}{\partial \boldsymbol{\eta}}\right)\right]=\frac{\partial}{\partial \boldsymbol{\eta}^{\prime}}\left[\operatorname{vec}\left(\boldsymbol{z}_{h} \boldsymbol{\beta}^{\prime}\right) \frac{1}{m_{h}}\right]=-\frac{1}{m_{h}^{2}} \operatorname{vec}\left(\boldsymbol{z}_{h} \boldsymbol{\beta}^{\prime}\right) \boldsymbol{z}_{h}^{\prime} .
\end{aligned}
$$

Then, using the first and the second derivatives of $\log p(\boldsymbol{\eta} \mid \cdots)$, we can obtain the mode of $\log p(\boldsymbol{\eta} \mid \cdots)$, say $\hat{\boldsymbol{\eta}}$. Now, define the tailored proposal density as follows:

$$
q\left(\boldsymbol{\eta}^{\prime}, \boldsymbol{\eta}\right)=q(\boldsymbol{\eta})=f_{M t}(\boldsymbol{\eta} \mid \hat{\boldsymbol{\eta}}, \boldsymbol{S}, \nu) \propto\left[1+\frac{1}{\nu}(\boldsymbol{\eta}-\hat{\boldsymbol{\eta}})^{\prime} \boldsymbol{S}^{-1}(\boldsymbol{\eta}-\hat{\boldsymbol{\eta}})\right]^{-(\nu+K) / 2} .
$$

where $\nu$ is adjustable constant ${ }^{21}$ and

$$
\boldsymbol{S}=\left[-\left.\frac{\partial^{2} \log p(\boldsymbol{\eta} \mid \cdots)}{\partial \boldsymbol{\eta} \partial \boldsymbol{\eta}^{\prime}}\right|_{\boldsymbol{\eta}=\hat{\boldsymbol{\eta}}}\right]^{-1}
$$

provided $\boldsymbol{S}$ is positive definite. Thus, we have the following M-H algorithm with the tailored proposal density:

1. At the $(t+1)$ th iteration, given the current sample $\boldsymbol{\eta}^{(t)}$, sample $\boldsymbol{\eta}$ from the proposal density $q(\boldsymbol{\eta})$.

2. Generate $u \sim \mathrm{U}(0,1)$, the uniform distribution on $(0,1)$ and take

$$
\boldsymbol{\eta}^{(t+1)}= \begin{cases}\boldsymbol{\eta} & \text { if } u<\min \left\{\frac{\mathrm{e}^{\log p(\boldsymbol{\eta} \mid \cdots)} q\left(\boldsymbol{\eta}^{(t)}\right)}{\mathrm{e}^{\log p\left(\boldsymbol{\eta}^{(t)} \mid \cdots\right)} q(\boldsymbol{\eta})}, 1\right\} \\ \boldsymbol{\eta}^{(t)} & \text { otherwise. }\end{cases}
$$

\footnotetext{
${ }^{21} \operatorname{In}$ Section 4 , we set $\nu=7$.
} 
TABLE 1

Number of zero expenditures

\begin{tabular}{lr}
\hline Number of households & 692 \\
\hline (1) Food & 7 \\
(2) Housing & 278 \\
(3) Fuel & 8 \\
(4) Furniture & 359 \\
(5) Clothing & 200 \\
(6) Medical & 253 \\
(7) Transport & 7 \\
(8) Others & 11 \\
\hline
\end{tabular}


TABLE 2

Posterior results for parameters

\begin{tabular}{lrrrrr}
\hline & \multicolumn{1}{c}{ mean } & sd & & mean & sd \\
\hline$\alpha_{1}$ & 0.56762 & 0.04249 & $\sigma_{11}$ & 0.0077473 & 0.0004278 \\
$\alpha_{2}$ & 0.21000 & 0.05159 & $\sigma_{21}$ & -0.0022755 & 0.0003394 \\
$\alpha_{3}$ & 0.31146 & 0.01720 & $\sigma_{31}$ & 0.0002357 & 0.0001129 \\
$\alpha_{4}$ & 0.00771 & 0.01416 & $\sigma_{41}$ & -0.0001544 & 0.0001045 \\
$\alpha_{5}$ & 0.04690 & 0.01910 & $\sigma_{51}$ & -0.0002563 & 0.0001277 \\
$\alpha_{6}$ & 0.03701 & 0.01564 & $\sigma_{61}$ & -0.0002448 & 0.0001096 \\
$\alpha_{7}$ & 0.09855 & 0.03524 & $\sigma_{71}$ & -0.0009417 & 0.0002488 \\
$\alpha_{8}$ & -0.27925 & 0.07585 & $\sigma_{22}$ & 0.0089570 & 0.0005241 \\
$\beta_{1}$ & -0.07066 & 0.00925 & $\sigma_{32}$ & -0.0003286 & 0.0001245 \\
$\beta_{2}$ & -0.01635 & 0.01113 & $\sigma_{42}$ & 0.0003557 & 0.0001041 \\
$\beta_{3}$ & -0.04969 & 0.00358 & $\sigma_{52}$ & -0.0000398 & 0.0001294 \\
$\beta_{4}$ & 0.00487 & 0.00309 & $\sigma_{62}$ & 0.0001045 & 0.0001127 \\
$\beta_{5}$ & -0.00040 & 0.00413 & $\sigma_{72}$ & -0.0006200 & 0.0002661 \\
$\beta_{6}$ & -0.00148 & 0.00339 & $\sigma_{33}$ & 0.0010774 & 0.0000597 \\
$\beta_{7}$ & 0.00153 & 0.00765 & $\sigma_{43}$ & 0.0000586 & 0.0000389 \\
$\beta_{8}$ & 0.13218 & 0.01580 & $\sigma_{53}$ & 0.0000868 & 0.0000477 \\
$\mu$ & 5.53810 & 0.01371 & $\sigma_{63}$ & 0.0000045 & 0.0000389 \\
$\tau$ & 0.12885 & 0.00697 & $\sigma_{73}$ & -0.0000986 & 0.0000916 \\
$\omega$ & 0.02396 & 0.01441 & $\sigma_{44}$ & 0.0006862 & 0.0000495 \\
$p_{1}$ & 0.97847 & 0.00542 & $\sigma_{54}$ & 0.0000552 & 0.0000405 \\
$p_{2}$ & 0.67823 & 0.01340 & $\sigma_{64}$ & 0.0000292 & 0.0000341 \\
$p_{3}$ & 0.98663 & 0.00472 & $\sigma_{74}$ & -0.0000807 & 0.0000789 \\
$p_{4}$ & 0.53905 & 0.01992 & $\sigma_{55}$ & 0.0012141 & 0.0000816 \\
$p_{5}$ & 0.73317 & 0.01064 & $\sigma_{65}$ & 0.0000980 & 0.0000440 \\
$p_{6}$ & 0.67793 & 0.02160 & $\sigma_{75}$ & -0.0001046 & 0.0001033 \\
$p_{7}$ & 0.98663 & 0.00274 & $\sigma_{66}$ & 0.0008488 & 0.0000669 \\
$p_{8}$ & 0.93285 & 0.00349 & $\sigma_{76}$ & 0.0001404 & 0.0000836 \\
$\eta_{1}$ & 0.36949 & 0.08494 & $\sigma_{77}$ & 0.0053424 & 0.0002893 \\
\hline & & & & &
\end{tabular}

Notes: 'mean' and 'sd' denote the posterior mean and posterior standard deviation. 
TABLE 3

Posterior results for elasticities

\begin{tabular}{lcc}
\hline & mean & sd \\
\hline Food & 0.6982 & 0.0396 \\
Housing & 0.8742 & 0.0857 \\
Fuel & 0.3554 & 0.0463 \\
Furniture & 1.1588 & 0.1006 \\
Clothing & 0.9912 & 0.0929 \\
Medical & 0.9504 & 0.1131 \\
Transport & 1.0142 & 0.0712 \\
Others & 1.3820 & 0.0457 \\
\hline
\end{tabular}

Notes: 'mean' and 'sd' denote the posterior mean and posterior standard deviation. 
TABLE 4

Posterior results for inequality measures

\begin{tabular}{lcccccc}
\hline & \multicolumn{3}{c}{ data } & & \multicolumn{2}{c}{ posterior } \\
\cline { 2 - 4 } \cline { 6 - 7 } & raw & p.c. & e.s. & & mean & sd \\
\hline$I_{\text {Gini }}$ & 0.2214 & 0.2640 & 0.2271 & & 0.2097 & 0.00377 \\
$I_{G E}(-1)$ & 0.0916 & 0.1273 & 0.0956 & & 0.0714 & 0.00259 \\
$I_{G E}(0)$ & 0.0825 & 0.1135 & 0.0858 & & 0.0705 & 0.00237 \\
$I_{G E}(1)$ & 0.0850 & 0.1168 & 0.0882 & & 0.0751 & 0.00239 \\
$I_{G E}(2)$ & 0.0993 & 0.1364 & 0.1022 & & 0.0878 & 0.00266 \\
\hline
\end{tabular}

Notes: 'raw,' 'p.c.' and 'e.s.' denote the inequality measures based on the total expenditure, the per capita total expenditure, and the total expenditure deflated by posterior equivalence scale, respectively. 'mean' and 'sd' denote the posterior mean and posterior standard deviation. 
TABLE 5

Classification of goods and number of zero expenditures*

\begin{tabular}{llll}
\hline$M=5$ & $M=6$ & $M=7$ & $M=8$ \\
\hline Food & Food & Food & Food \\
7 & 7 & 7 & 7 \\
\hline Housing & Housing & Housing & Housing \\
148 & 278 & 278 & 278 \\
\cline { 2 - 4 } & Furniture & Furniture & Furniture \\
& 359 & 359 & 359 \\
\hline Fuel & Fuel & Fuel & Fuel \\
8 & 8 & 8 & 8 \\
\hline Clothing & Clothing & Clothing & Clothing \\
200 & 200 & 200 & 200 \\
\hline Others & Others & Medical & Medical \\
1 & 1 & 253 & 253 \\
\cline { 2 - 4 } & & Others & Transport \\
& & 1 & 7 \\
\cline { 3 - 4 } & & & Others \\
\hline
\end{tabular}

Notes: ${ }^{*}$ The figures in lower row denote the number of zero expenditures in the item. 
TABLE 6

Number of children and age grouping

\begin{tabular}{crrrrr}
\hline \# of children \age & $0 \sim 6$ & $7 \sim 12$ & $0 \sim 12$ & $13 \sim 18$ & $0 \sim 18$ \\
\hline 0 & $312^{*}$ & 368 & 129 & 571 & 99 \\
1 & 236 & 201 & 223 & 82 & 173 \\
2 & 133 & 113 & 259 & 35 & 296 \\
3 & 11 & 9 & 73 & 3 & 105 \\
4 & 0 & 1 & 7 & 1 & 17 \\
5 & 0 & 0 & 1 & 0 & 2 \\
\hline sum & $535^{\dagger}$ & 458 & 993 & 165 & 1,158 \\
\hline
\end{tabular}

Notes: '\# of children' denotes the number of children in a household. 'age' denotes the age group of children. For example, ' $0 \sim 6$ 'means the age group of children aged 0 to 6 years. The figure of ${ }^{*}$ denotes the number of households. The figure of ${ }^{\dagger}$ denotes the number of children corresponding to the age group in the data. 
TABLE 7

Posterior means of purchasing probabilities

\begin{tabular}{|c|c|c|c|c|c|c|c|c|}
\hline & \multicolumn{4}{|c|}{$M=5$} & \multicolumn{4}{|c|}{$M=6$} \\
\hline & $K=1$ & $K=2$ & $K=3$ & data & $K=1$ & $K=2$ & $K=3$ & data \\
\hline Food & 0.991 & 0.986 & 0.989 & 0.990 & 0.986 & 0.986 & 0.985 & 0.990 \\
\hline Housing & 0.771 & 0.786 & 0.788 & 0.786 & 0.615 & 0.637 & 0.602 & 0.598 \\
\hline Fuel & 0.988 & 0.987 & 0.987 & 0.988 & 0.987 & 0.988 & 0.988 & 0.988 \\
\hline Furniture & & & & & 0.536 & 0.538 & 0.543 & 0.481 \\
\hline Clothing & 0.741 & 0.733 & 0.748 & 0.711 & 0.724 & 0.738 & 0.735 & 0.711 \\
\hline Medical & & & & & & & & \\
\hline Transport & & & & & & & & \\
\hline \multirow[t]{3}{*}{ Others } & 0.969 & 0.987 & 0.964 & 0.999 & 0.978 & 0.970 & 0.949 & 0.999 \\
\hline & \multicolumn{4}{|c|}{$M=7$} & \multicolumn{4}{|c|}{$M=8$} \\
\hline & $K=1$ & $K=2$ & $K=3$ & data & $K=1$ & $K=2$ & $K=3$ & data \\
\hline Food & 0.987 & 0.984 & 0.981 & 0.990 & 0.978 & 0.983 & 0.978 & 0.990 \\
\hline Housing & 0.668 & 0.683 & 0.663 & 0.598 & 0.678 & 0.668 & 0.642 & 0.598 \\
\hline Fuel & 0.987 & 0.988 & 0.985 & 0.988 & 0.987 & 0.988 & 0.988 & 0.988 \\
\hline Furniture & 0.550 & 0.548 & 0.533 & 0.481 & 0.539 & 0.540 & 0.530 & 0.481 \\
\hline Clothing & 0.729 & 0.720 & 0.739 & 0.711 & 0.733 & 0.729 & 0.725 & 0.711 \\
\hline Medical & 0.678 & 0.678 & 0.701 & 0.634 & 0.678 & 0.679 & 0.662 & 0.634 \\
\hline Transport & & & & & 0.987 & 0.989 & 0.981 & 0.990 \\
\hline Others & 0.927 & 0.953 & 0.953 & 0.999 & 0.933 & 0.936 & 0.949 & 0.984 \\
\hline
\end{tabular}

Notes: 'data' denotes $1-\frac{1}{H} \sum D_{h i}$. 
TABLE 8

Posterior means of $\boldsymbol{\eta}$ in equivalence scales

\begin{tabular}{llllllll}
\hline & \multicolumn{3}{c}{$M=5$} & & & \multicolumn{3}{c}{$M=6$} \\
\cline { 2 - 4 } \cline { 6 - 8 } & $K=1$ & $K=2$ & $K=3$ & & $K=1$ & $K=2$ & $K=3$ \\
\hline$\eta_{1}$ & 0.4094 & 0.3641 & 0.2152 & & 0.3320 & 0.2799 & 0.1823 \\
$\eta_{2}$ & & 0.9459 & 0.5235 & & 0.7240 & 0.3815 \\
$\eta_{3}$ & & & 0.8558 & & & 0.6857 \\
\hline \hline & & $M=7$ & & & & $M=8$ & \\
\cline { 2 - 3 } \cline { 6 - 8 } & $K=1$ & $K=2$ & $K=3$ & & $K=1$ & $K=2$ & $K=3$ \\
\hline$\eta_{1}$ & 0.3415 & 0.3440 & 0.2013 & & 0.3695 & 0.3010 & 0.2319 \\
$\eta_{2}$ & & 0.8799 & 0.4073 & & 0.8242 & 0.4902 \\
$\eta_{3}$ & & & 0.7520 & & & 0.8689 \\
\hline
\end{tabular}


TABLE 9

Posterior means of elasticities

\begin{tabular}{|c|c|c|c|c|c|c|}
\hline & \multicolumn{3}{|c|}{$M=5$} & \multicolumn{3}{|c|}{$M=6$} \\
\hline & $K=1$ & $K=2$ & $K=3$ & $K=1$ & $K=2$ & $K=3$ \\
\hline Food & 0.6705 & 0.6206 & 0.5984 & 0.6843 & 0.6522 & 0.6213 \\
\hline Housing & 0.9667 & 1.0725 & 1.0979 & 0.8250 & 0.8208 & 0.8642 \\
\hline Fuel & 0.3442 & 0.3453 & 0.3478 & 0.3293 & 0.3283 & 0.3149 \\
\hline Furniture & & & & 0.9858 & 1.0842 & 1.1140 \\
\hline Clothing & 0.8398 & 0.8503 & 0.8700 & 0.8500 & 0.9107 & 0.9262 \\
\hline \multirow{2}{*}{\multicolumn{7}{|c|}{$\begin{array}{l}\text { Medical } \\
\text { Transport }\end{array}$}} \\
\hline & & & & & & \\
\hline \multirow[t]{3}{*}{ Others } & 1.2903 & 1.2758 & 1.2843 & 1.3163 & 1.3250 & 1.3339 \\
\hline & \multicolumn{3}{|c|}{$M=7$} & \multicolumn{3}{|c|}{$\overline{\bar{n}} M=8$} \\
\hline & $K=1$ & $K=2$ & $K=3$ & $K=1$ & $K=2$ & $K=3$ \\
\hline Food & 0.6955 & 0.6584 & 0.6254 & 0.6982 & 0.6455 & 0.6081 \\
\hline Housing & 0.8547 & 0.9501 & 0.9183 & 0.8742 & 0.9401 & 1.0452 \\
\hline Fuel & 0.3409 & 0.3483 & 0.3274 & 0.3554 & 0.3460 & 0.3503 \\
\hline Furniture & 1.1686 & 1.0164 & 1.1152 & 1.1588 & 1.1142 & 1.1199 \\
\hline Clothing & 0.9446 & 0.9591 & 1.0036 & 0.9912 & 1.0219 & 1.0742 \\
\hline Medical & 1.0472 & 1.0829 & 1.0504 & 0.9504 & 1.0620 & 1.0881 \\
\hline Transport & & & & 1.0142 & 1.0183 & 1.0179 \\
\hline Others & 1.3090 & 1.2996 & 1.3190 & 1.3820 & 1.3815 & 1.3544 \\
\hline
\end{tabular}


TABLE 10

Inequality measures for goods and age groups

\begin{tabular}{|c|c|c|c|c|c|c|c|}
\hline & & & $I_{\text {Gini }}$ & $I_{G E}(-1)$ & $I_{G E}(0)$ & $I_{G E}(1)$ & $\overline{I_{G E}(2)}$ \\
\hline & & raw & 0.2214 & 0.0916 & 0.0825 & 0.0850 & 0.0993 \\
\hline & & p.c. & 0.2640 & 0.1273 & 0.1135 & 0.1168 & 0.1364 \\
\hline \multirow{6}{*}{$M=5$} & \multirow[t]{2}{*}{$K=1$} & e.s. & 0.2289 & 0.0971 & 0.0871 & 0.0895 & 0.1037 \\
\hline & & mean & 0.2153 & 0.0759 & 0.0745 & 0.0792 & 0.0926 \\
\hline & \multirow[t]{2}{*}{$K=2$} & e.s. & 0.2294 & 0.0959 & 0.0867 & 0.0893 & 0.1035 \\
\hline & & mean & 0.2175 & 0.0767 & 0.0754 & 0.0800 & 0.0931 \\
\hline & \multirow[t]{2}{*}{$K=3$} & e.s. & 0.2291 & 0.0966 & 0.0869 & 0.0892 & 0.1027 \\
\hline & & mean & 0.2135 & 0.0746 & 0.0732 & 0.0776 & 0.0900 \\
\hline \multirow{6}{*}{$M=6$} & \multirow[t]{2}{*}{$K=1$} & e.s. & 0.2254 & 0.0944 & 0.0847 & 0.0871 & 0.1010 \\
\hline & & mean & 0.2144 & 0.0758 & 0.0742 & 0.0790 & 0.0926 \\
\hline & \multirow[t]{2}{*}{$K=2$} & e.s. & 0.2238 & 0.0917 & 0.0830 & 0.0856 & 0.0992 \\
\hline & & mean & 0.2098 & 0.0716 & 0.0708 & 0.0759 & 0.0898 \\
\hline & \multirow[t]{2}{*}{$K=3$} & e.s. & 0.2230 & 0.0916 & 0.0827 & 0.0851 & 0.0983 \\
\hline & & mean & 0.2072 & 0.0700 & 0.0690 & 0.0735 & 0.0853 \\
\hline \multirow{6}{*}{$M=7$} & \multirow[t]{2}{*}{$K=1$} & e.s. & 0.2258 & 0.0947 & 0.0850 & 0.0874 & 0.1013 \\
\hline & & mean & 0.2115 & 0.0726 & 0.0717 & 0.0765 & 0.0896 \\
\hline & \multirow[t]{2}{*}{$K=2$} & e.s. & 0.2278 & 0.0947 & 0.0857 & 0.0883 & 0.1023 \\
\hline & & mean & 0.2122 & 0.0731 & 0.0720 & 0.0767 & 0.0897 \\
\hline & \multirow[t]{2}{*}{$K=3$} & e.s. & 0.2246 & 0.0927 & 0.0837 & 0.0861 & 0.0994 \\
\hline & & mean & 0.2087 & 0.0707 & 0.0702 & 0.0753 & 0.0887 \\
\hline \multirow{6}{*}{$M=8$} & \multirow[t]{2}{*}{$K=1$} & e.s. & 0.2271 & 0.0956 & 0.0858 & 0.0882 & 0.1022 \\
\hline & & mean & 0.2097 & 0.0714 & 0.0705 & 0.0751 & 0.0878 \\
\hline & \multirow[t]{2}{*}{$K=2$} & e.s. & 0.2256 & 0.0929 & 0.0841 & 0.0867 & 0.1005 \\
\hline & & mean & 0.2079 & 0.0691 & 0.0689 & 0.0738 & 0.0864 \\
\hline & \multirow[t]{2}{*}{$K=3$} & e.s. & 0.2284 & 0.0959 & 0.0864 & 0.0887 & 0.1023 \\
\hline & & mean & 0.2115 & 0.0725 & 0.0716 & 0.0763 & 0.0889 \\
\hline
\end{tabular}

Notes: 'raw,' 'p.c.' and 'e.s.' denote the inequality measures based on the total expenditure, the per capita total expenditure, and the total expenditure deflated by posterior equivalence scale, respectively. 'mean' denotes the posterior mean. 